J. Nonlinear Var. Anal. 3 (2019), No. 1, pp. 1-3

Available online at http://jnva.biemdas.com

https://doi.org/10.23952/jnva.3.2019.1.01

\title{
EDITORIAL
}

\section{A SPECIAL ISSUE ON RECENT PROGRESS IN NONLINEAR AND VARIATIONAL ANALYSIS DEDICATED TO WATARU TAKAHASHI'S 75TH BIRTHDAY}

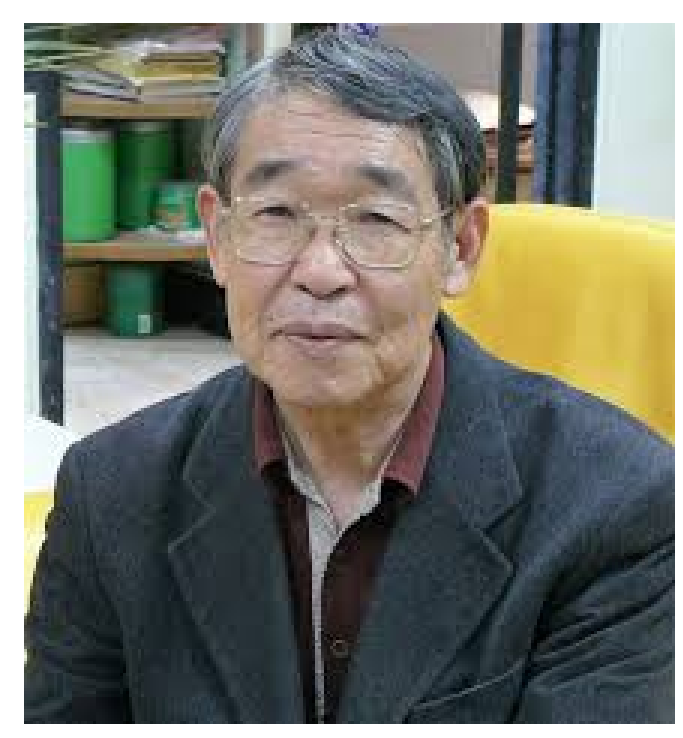

This special issue of Recent Progress in Nonlinear and Variational Analysis is edited in honor of Professor Wataru Takahashi for his substantial contribution to nonlinear and variational analysis and for his scientific leadership.

Professor Wataru Takahashi is an Emeritus Professor of the Department of Mathematical and Computing Sciences at Tokyo Institute of Technology in Japan, a Professor of the Keio Research and Education Center for Natural Sciences at Keio University in Japan and a Professor of the Research Center for Interneural Computing at China Medical University Hospital at China Medical University in Taiwan. He is also one of the world's leading researchers in fixed point theory and nonlinear functional analysis.

Professor Wataru Takahashi obtained his B.S. (in 1966) from Yokohama National University and D.Sc. (in 1971) from Tokyo Institute of Technology and was a student of Professor Toshio Aoki and Professor Hisaharu Umegaki. Professor Wataru Takahashi is also influenced by Professor Ky Fan. With the belief that "nonlinear functional analysis is not a subsidiary of linear functional analysis and fixed 
point property is a universal concept in nonlinear analysis", Professor Wataru Takahashi started and initiated his long phase of the development of nonlinear analysis and convex analysis and student training.

Professor Wataru Takahashi is known as one of the highly cited researchers in the world from 2014 to 2018 (MathSciNet Citations 8757). His most influential papers are "Fixed point theorem for amenable semigroup of nonexpansive mappings (Kodai Math. Sem. Rep. 21-4, 1969, 383-386)", describing a fixed point theorem for nonexpansive semigroups of nonexpansive mappings in a Banach space and combining notions from Harmonic Analysis and Nonlinear Analysis; "A convexity of metric space and nonexpansive mappings I (Kodai Math. Sem. Rep. 22-2, 1970, 142-149)", introducing the convexity in metric spaces; "Nonlinear variational inequalities and fixed point theorem (J. Math. Soc. Japan 28-1, 1976, 168-181)", proving Fan's inequality theorem independently and obtaining many important results by using the theorem; "A nonlinear ergodic theorem for an amenable semigroup of nonexpansive mappings in a Hilbert space (Proc. Amer. Math. Soc. 81-2, 1981, 253-256)", introducing a mean value for abstract semigroups by using the Riesz theorem and proving the first nonlinear ergodic theorem for noncommutative semigroups of nonexpansive mappings in a Hilbert space; "Existence theorems generalizing fixed point theorems for multivalued mappings (in Fixed Point Theory and its Applications, J. B. Baillon and M. Thera Eds., Pitman Res. Notes in Math. Ser. 252, 1991, 397-406)”, proving a nonconvex minimization theorem in a complete metric space and giving simpler proofs of Caristi's fixed point theorem and Ekeland's variational principle. He and his collaborators proved ultimate fixed point and mean convergence theorems for noncommutative semigroups of nonexpansive mappings; see "Invariant submeans and semigroups of nonexpansive mappings on Banach spaces with normal structure (J. Funct. Anal. 142-1, 1996, 79-88)" and "Existence of nonexpansive retractions for amenable semigroups of nonexpansive mappings and nonlinear ergodic theorems in Banach spaces (J. Funct. Anal. 161-1, 1999, 62-75)". He and his collaborators also made a break through in proximal algorithms in Banach spaces; see "Strong convergence of a proximal-type algorithm in a Banach space (SIAM J. Optim. 13-2, 2002, 938-945)" and "Weak and strong convergence theorems for maximal monotone operators in a Banach space (Set-Valued Anal. 12-4, 2004, 417-429)". Furthermore, he and his collaborators introduced the hybrid method and the shrinking projection method and proved important results which have been referred many authors; see "Strong convergence theorems for nonexpansive mapping and nonexpansive semigroups (J. Math. Anal. Appl. 279-2, 2003, 372-379)" and "Strong convergence theorems by hybrid methods for families of nonexpansive mappings in Hilbert spaces (J. Math. Anal. Appl. 341-1, 2008, 276-286)". Recently, Professor Wataru Takahashi solved the split common null point promlem and the split common fixed point problem in two Banach spaces which are first outside Hilbert spaces; see "The split common null point problem in two Banach spaces (J. Nonlinear Convex Anal. 16-12, 2015, 23432350)" and "The split common fixed point problem and strong convergence theorems by hybrid methods in two Banach spaces (J. Nonlinear Convex Anal. 17-6, 2016), 1051-1067)".

Professor Wataru Takahashi is renowned as an energetic teacher and educator. He has supervised more than $40 \mathrm{PhD}$ students from five countries. In addition to his many fundamental papers, he also serves as the chief editor of the journal Journal of Nonlinear and Convex Analysis and published a research book titled "Nonlinear Functional Analysis", published by Yokohama Publishers, 2000. We sincerely wish his versatility and his insight will achieve an unpredictable phase. 


\section{Mayumi Hojo \\ College of Engineering}

Shibaura Institute of Technology, 307 Fukasaku, Minuma-ku, Saitama 337-8570, Japan

E-mail address: mayumi-h@shibaura-it.ac.jp

Xiaolong Qin
Institute of Fundamental and Frontier Sciences
University of Electronic Science and Technology of China, Chengdu 610054, China
E-mail address: qxlxajh@ 163.com
Jen-Chih Yao
Research Center for Interneural Computing
China Medical University Hospital
China Medical University, Taichung, Taiwan
E-mail address: yaojc@ mail.cmu.edu.tw

